\title{
Caracterización Morfométrica del Reborde Anterior de la Maxila Mediante Tomografía Computarizada Cone-Beam
}

\author{
Morphometric Characterization of Anterior Maxillary Alveolar \\ Ridge by Cone-Beam Computed Tomography
}

Ramón Fuentes*; Pablo Navarro**; Carlos Salamanca*; Mario Cantín"****; Ivonne Garay* \& Tania Flores ${ }^{* * * * *}$

FUENTES, R.; NAVARRO, P.; SALAMANCA, C.; CANTíN, M.; GARAY, I. \& FLORES, T. Caracterización morfométrica del reborde anterior de la maxila mediante tomografía computarizada Cone-Beam. Int. J. Morphol., 32(2):493-498, 2014.

RESUMEN: Las imágenes obtenidas por Tomografía Computarizada Cone-Beam (TCCB) permiten determinar las relaciones de estructuras anatómicas tridimensionalmente y superar limitaciones de la radiografía convencional. El objetivo de este estudio fue describir las características morfométricas de la zona anterior del maxilar y su relación con los dientes canino y premolares mediante exámenes de TCCB. Se realizó un estudio descriptivo sobre 50 imágenes de TCCB siguiendo una sistemática de observación tridimensional se ubicaron puntos anatómicos entre dientes y paredes del seno maxilar, y se trazaron tangentes para su medición. Se calculo la Media y DE para cada parámetro, y la normalidad de la distribución se comprobó mediante la prueba de Kolmogorov-Smirnov. Los datos fueron comparados con la prueba ANOVA, con una valor $\mathrm{p}<0,05$ como significativo. El análisis de la relación entre los dientes y la pared anterior del seno maxilar, mostró que en un $80 \%$ de los casos se relacionó con el canino y $20 \%$ con el primer premolar. La menor altura

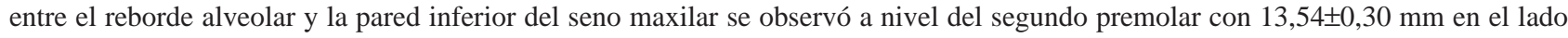
derecho y 14,65 $\pm 0,30 \mathrm{~mm}$ en el izquierdo. Se registraron diferencias significativas en relación a las mediciones morfométricas y la variable rango etáreo. Los datos obtenidos en este estudio complementan las características morfométricas de la zona anterior del maxilar, lo que permite ejecutar tratamientos de implantes bajo una base morfológica científica y minimizando al máximo los riesgos de fracaso o daño a las estructuras anatómicas relacionadas.

PALABRAS CLAVE: Seno maxilar; Implantes dentales; Diente canino; Primer premolar maxilar.

\section{INTRODUCCIÓN}

Los primeros premolares maxilares, son dientes que frecuentemente presentan fracturas radiculares, lo que conlleva a su indicación de extracción. Su posterior rehabilitación, es importante por ser un sector estéticamente visible (Nothdurft et al., 2008). Sin embargo, en general no sólo se pierde el diente, ya que la tabla ósea alveolar bucal del hueso maxilar con frecuencia también se fractura y colapsa durante la extracción dentaria debido a sus características anatómicas (Leblebicioglu et al., 2007), o bien, la altura y grosor de las tablas óseas disminuyen por la falta de estímulo o transmisión de fuerzas. Swart \& van Niekerk (2008) sugieren que la opción más aceptable de tratamiento para reemplazar un premolar perdido es la colocación de implante, pero su colocación en un sitio de extracción reciente, no evita la remodelación y reabsorción ósea del reborde alveolar en su totalidad (Araujo \& Lindhe, 2005).
Actualmente, la necesidad de mayor precisión en los diagnósticos y tratamientos dentales ha aumentado, lo que ha puesto de manifiesto las limitaciones de las radiografías dentales y las tomografías computarizadas (TC) convencionales respecto a su capacidad para proporcionar información cualitativa, cuantitativa y tridimensional precisa, con desventajas como la distorsión, poca definición y falta de referencia a estructuras anatómicas adyacentes (BarrachinaMataix et al., 2002, Bergkvist et al., 2005; Arana-Fernández et al., 2006; Mantini \& Ripani, 2009).

Según Ausbruch et al. (2010) la radiografía panorámica es el estudio más solicitado por los implantólogos, pero sólo por la cantidad de información que brinda y no por la precisión que ofrece, ya que proporciona imágenes no uniformes. La prescripción sólo de la radiografía panorámica

* Departamento de Odontología Integral, Facultad de Odontología Universidad de La Frontera, Temuco, Chile.

** Departamento de Matemáticas y Estadísticas, Universidad de La Frontera, Temuco, Chile.

**** Centro de Investigación en Ciencias Biomédicas, Universidad Autónoma de Chile, Temuco, Chile.

***** Programa de Magíster en Odontología, Facultad de Odontología, Universidad de La Frontera, Temuco, Chile. 
para tomar medidas, está completamente contraindicada, ya que se ha demostrado que los niveles de magnificación no son uniformes, y es imposible transportar las medidas de la misma a la boca del paciente. Mozzo et al. (1998) compararon la utilidad del sistema de TC Cone-Beam (TCCB) y la TC convencional para la planificación de implantes en áreas del complejo maxilofacial, sugiriendo que la TCCB permite una mejor visualización de las estructuras maxilofaciales.

Aunque existe evidencia de mediciones sobre estructuras dentarias como puntos de referencia anatómica, generalmente no se relacionan con la morfometría ósea en altura (Fuentes et al., 2011; Figún et al., 2002), sobretodo en relción a la pared anterior del seno maxilar, por lo que es necesario obtener mayor información de las relaciones entre las raíces dentarias y el reborde alveolar en altura a través de imágenes 3D para estimar la morfometría ósea del maxila en el secto anterior.

El objetivo de este estudio fue describir las características morfométricas de la zona anterior de la maxila y su relación en altura con los dientes canino y premolares mediante exámenes de Tomografía Computarizada Cone-Beam.

\section{MATERIAL Y MÉTODO}

Se realizó un estudio descriptivo de corte transversal. Fueron analizados exámenes de TCCB atendidos en la Unidad de Radiología, Facultad de Odontología, Universidad de La Frontera (UFRO). El estudio fue aprobado por el Comité de Ética Científica de la UFRO (Protocolo No 72/2013).

Se realizó un muestreo por conveniencia, donde se incluyeron exámenes de TCCB tomados con una ventana de 120x90 mm pertenecientes a sujetos de ambos sexos, sin distinción étnica, y presencia de los dientes canino, primer (1PM) y segundo premolar (2PM) maxilar del lado derecho e izquierdo. Fueron excluidos exámenes TCCB de pacientes tratados o bajo tratamiento de radioterapia, ortodoncia, con presencia de coronas protésicas en los dientes canino, 1PM y 2PM o patologías óseas concomitantes.

Adquisición de las imágenes y mediciones. Todos los exámenes imagenológicos tridimensionales fueron adquiridos con el Tomógrafo PAX Zenith 3D (Vatech Co., Gyeonggi-Do, Korea). Los datos obtenidos se exportaron en formato DICOM al software EZ3D2009 (E-WOO Technology Co, Ltd. Korea), y se generaron las reconstrucciones con cortes de $1 \mathrm{~mm}$ de intervalo y $2 \mathrm{~mm}$ de grosor.

La medición morfométrica de las imágenes se realizó por dos examinadores calibrados, en los mismos cortes de las imagen. Para todas las observaciones y mediciones realizadas en el lado derecho e izquierdo, las imágenes de TCCB se orientaron con el software Ez3D2009 de la siguiente manera:

1. En una vista axial, se seleccionó un trazo curvo sobre la zona central de las caras oclusales de los dientes del hemiarco derecho (Fig. 1).

2. Se seleccionó el primer corte, en el que visualizó claramente la conjunción nasosinusal (Fig. 2).

3. Sobre la vista panorámica, se determinaron los límites de la imagen: el margen infraorbitario como límite superior, la línea oclusal como límite inferior, la pared lateral de la fosa nasal y pared posterior del seno maxilar homolateral como paredes laterales.

4. En el corte seleccionado se realizó un primer trazado tangente al punto más anterior del seno maxilar (tangente 1), localizable a nivel de la conjunción nasosinusal y asimismo paralelo a la línea mediana. Al mismo tiempo se registró el diente directamente relacionado con la porción más anterior del seno maxilar (tangente 1). Se trazó una segunda tangente paralela a la primera y por mesial del primer molar a fin de delimitar la zona de medición.

5. Sobre el canino y los premolares se determinaron puntos anatómicos y se midieron los trazos resultantes de sus uniones, respetando estrictamente el orden mencionado a continuación. Los puntos fueron i) Punto A (A): punto más apical del diente a medir, ii) Punto $\mathrm{P}(\mathrm{P})$ : punto localizado a nivel de la pared inferior del seno maxilar que se obtiene de la intersección de un trazo paralelo a la tangente 1 que pasa por el eje longitudinal de los dientes en estudio, iii) Punto $\mathrm{R}(\mathrm{R})$ : punto localizado por distal de los dientes y a nivel del reborde alveolar y iv) Límite amelocementario (LAC): punto localizado en la zona de unión cemento esmalte de los dientes y por distal de estos (Figs. 3 y 4 ).

6. Luego se determinaron trazos de medición: i) A-P: unión de punto A y P, ii) R-A: unión de punto R y A, iii) R-P: unión de punto R y P y iv) LAC-A: unión de punto LAC y A. Todas las mediciones se realizaron sobre los trazos, midiendo primero el lado derecho y luego el lado izquierdo. El resultado de las mediciones se registró en una tabla resumen diseñada para dicho efecto.

Análisis Estadístico. La estadística descriptiva se realizó en base al cálculo de la Media y DE. Para la estimación de la altura del reborde se utilizó Intervalos de Confianza del 95\%, de modo que, el verdadero promedio de las mediciones estuvo comprendido entre el limite superior e inferior de los valores de los trazos y que en 95 de 100 veces realizada la 


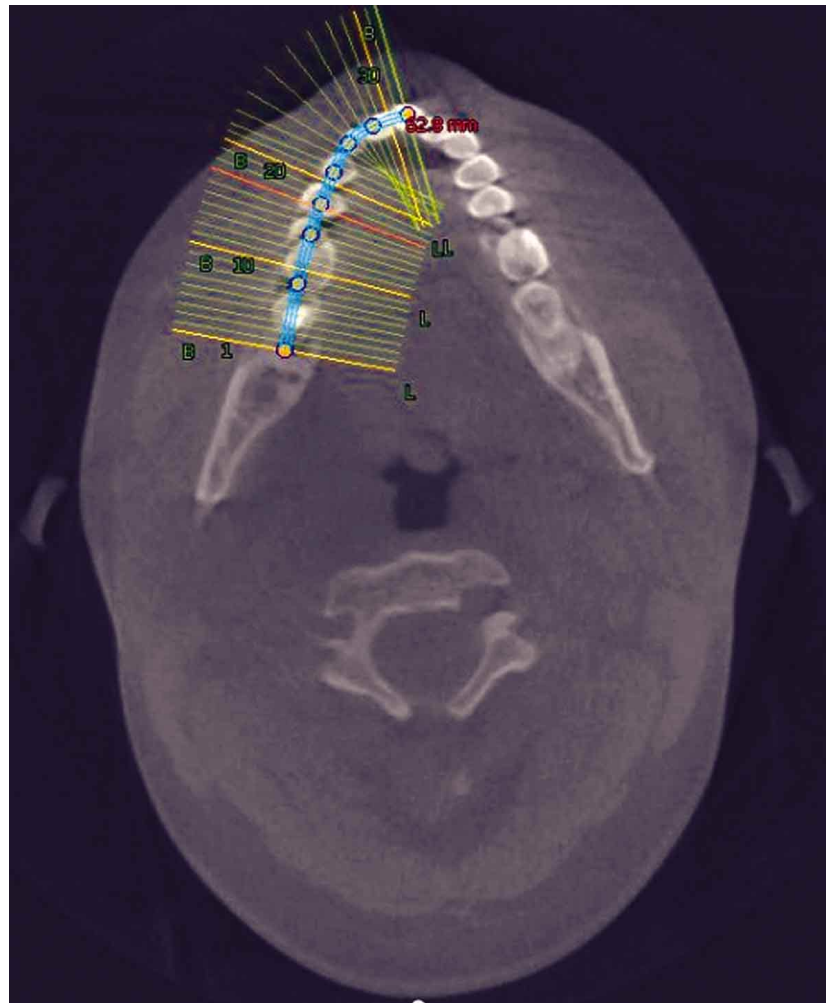

Fig. 1. Vista axial de cráneo y selección de trazo curvo sobre caras oclusales de dientes del hemiarco derecho.

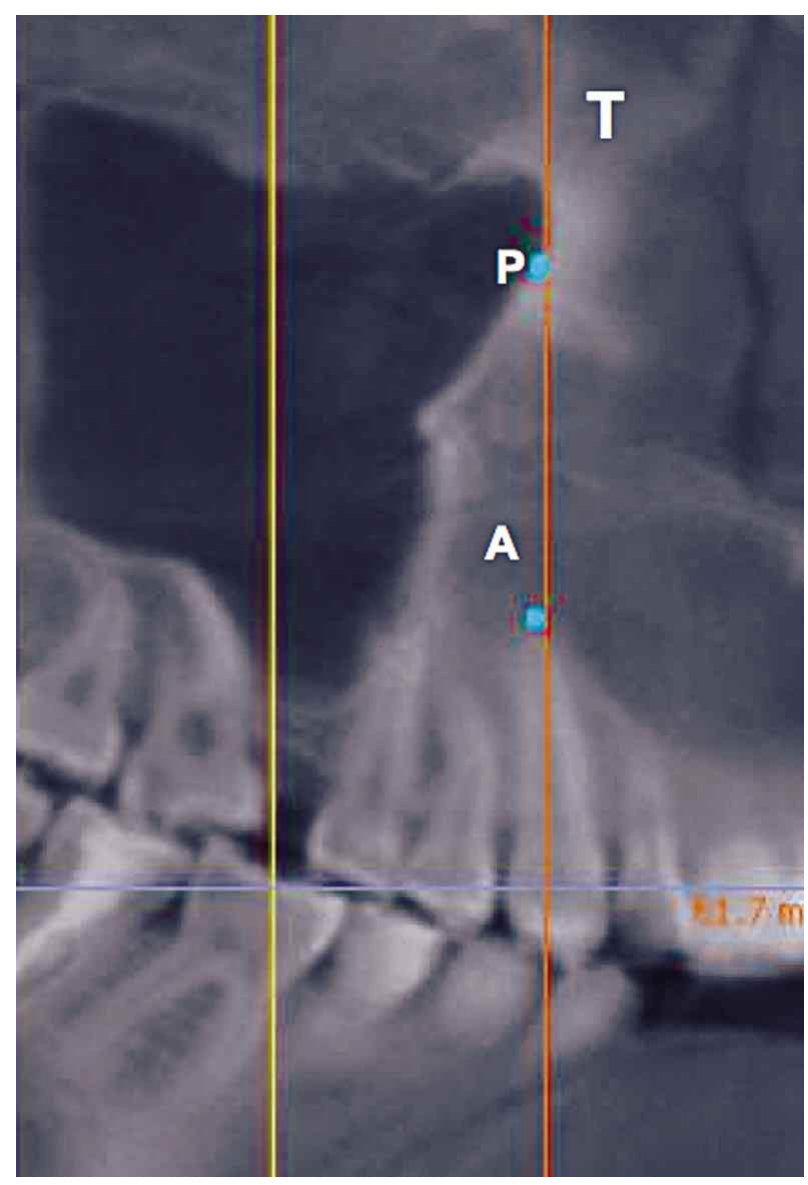

Fig. 3. Determinación de puntos A y P; T: tangente a pared anterior del seno maxilar.

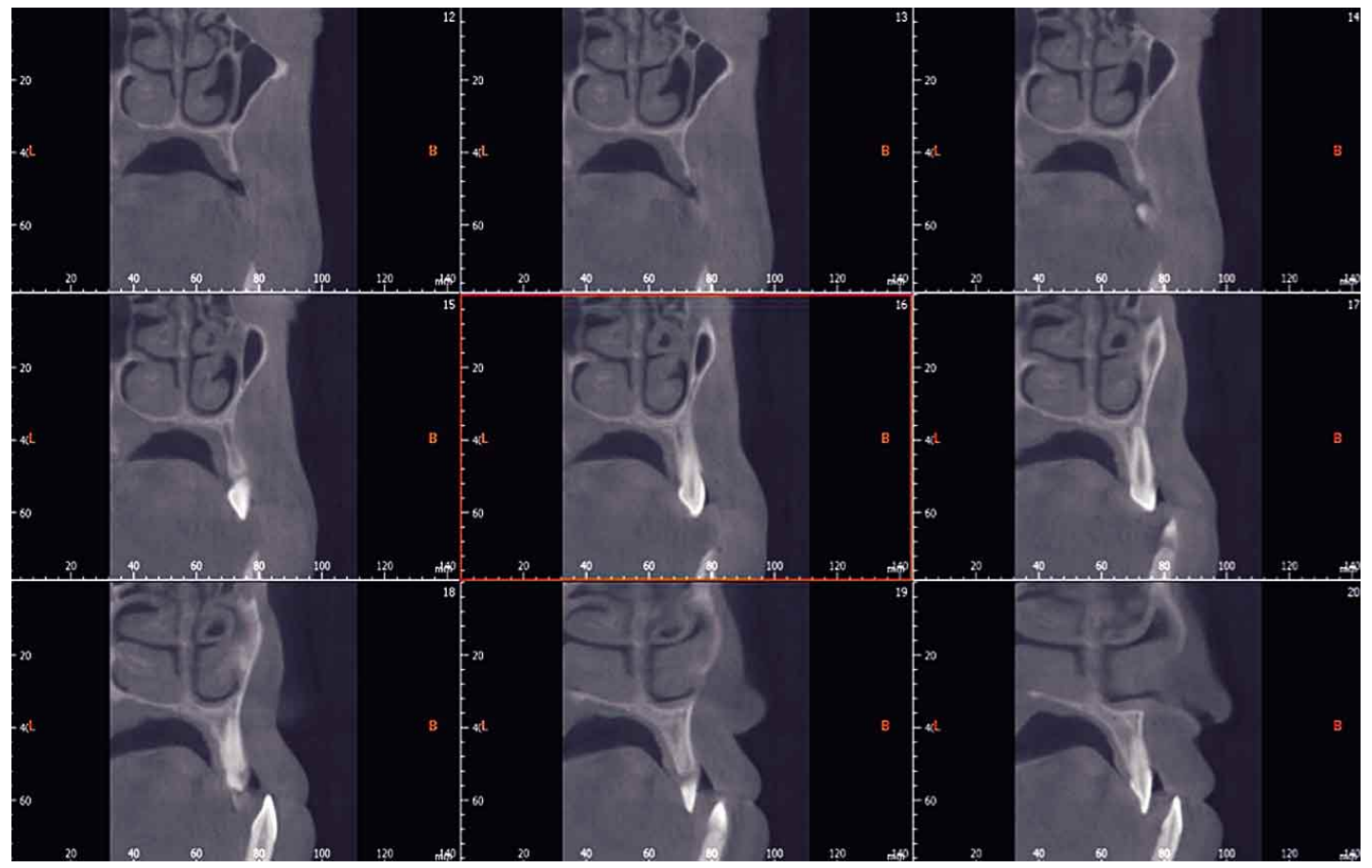

Fig. 2. Selección del corte de visualización de la conjunción nasosinusal. 


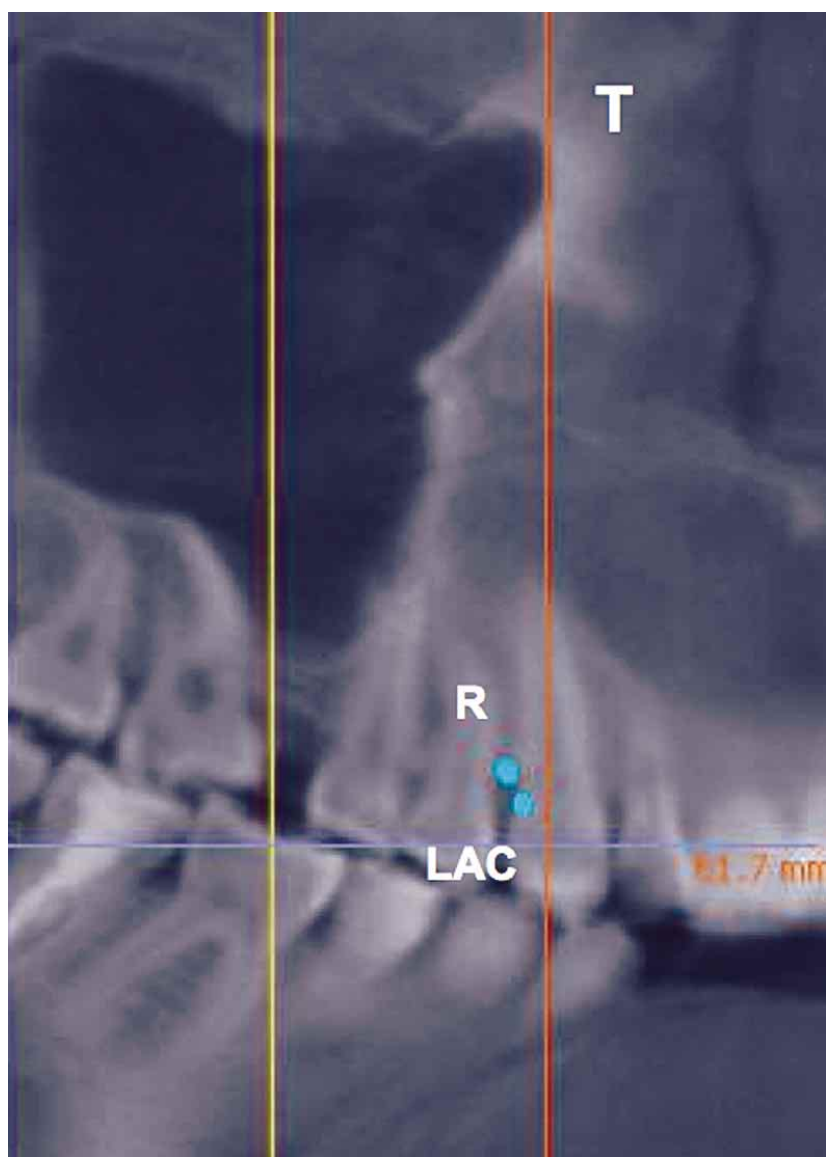

Fig. 4. Determinación de puntos R y LAC. T: tangente a pared anterior del seno maxilar.

prueba, el valor de las mediciones se encontró comprendido dentro de este intervalo. La normalidad de la distribución de las mediciones se comprobó mediante la prueba de Kolmogorov-Smirnov. Para la determinación de diferencias significativas en relación a la variabilidad entre los varios grupos de datos se utilizó la pruba ANOVA. En los casos en que la media de uno de los grupos fue diferente a las restantes, se utilizó la Prueba de Scheffé o Bonferroni, junto a la Prueba Chi Cuadrado de Pearson y la Prueba t-Student. El valor de $\mathrm{p}<0,05$ se consideró como umbral de la significación estadística.

\section{RESULTADOS}

Fueron estudiados un total de 50 exámenes TCCB, siendo 37 pertenecientes al sexo femenino $(74,0 \%)$ y 13 al sexo masculino $(26,0 \%)$. La edad de los pacientes fluctuaba entre 18 y 64 años, un $60 \%$ de los cuales se encontraba en el rango de entre 18 y 30 años.
El análisis de la relación entre los dientes y la tangente a la pared anterior del seno maxilar, mostró que en un $80 \%$ de los casos, la relación se observó con el dientecanino y en un $20 \%$ con el 1PM. En un $65 \%$ de los caninos, la relación se observó por mesial. Ambas variables (diente relacionado y zona de relación) se presentaron independientes del sexo, rango etáreo y hemiarco estudiado. A partir de las mediciones realizadas, fue posible generar la estimación de la altura del reborde y distancias de importancia clínica de la zona estudiada como se expresa en la Tabla I.

Se registraron diferencias significativas en relación a las mediciones de los trazos y la variable rango etáreo. En el lado izquierdo sobre el trazo A-P del canino, entre 18-30 y 31-40 años $(\mathrm{p}=0,009)$ y entre $41-50$ y $51-60$ años $(\mathrm{p}=0,001)$, un incremento de la diferencia a medida que aumentó la edad. En el mismo lado sobre el trazo R-A, se observó diferencia a nivel del 1PM, entre los rangos de 18-30 y 41-50 años $(\mathrm{p}=0,030)$ y entre $41-50$ y $51-60$ años $(\mathrm{p}=0,010)$. En el lado derecho, sobre el trazo A-P del 2PM, entre los rangos de 18-30 y 31-40 años, se observó un valor $\mathrm{p}=0,048$. En el mismo lado y sobre el trazo LAC-A del canino, se observaron diferencias entre los rangos de 18-30 y 51-60 años $(p=0,025)$. La consistencia interna entre los dos examinadores fue excelente.

\section{DISCUSIÓN}

Las imágenes obtenidas a través de los exámenes TCCB contribuyen a ampliar el conocimiento de zonas poco exploradas o caracterizadas por limitaciones técnicas de los imágenes en dos dimensiones, ya que la TCCB genera imágenes tridimensionales, lo que permite el estudio de estructuras anatómicas, parámetros morfológicos y distancias entre las estructuras de importancia en implantología (Oviedo \& Hernández, 2012), lo cual verificamos en el presente estudio, identificando dientes y límites del seno maxilar en el sector anterior de manera exacta; además, las mediciones obtenidas se corresponden a distancias de gran presición, lo que no sucede en radiografías panorámicas.

Debido a las altas necesidades estéticas del sector anterior del maxilar, la mayoría de las investigaciones han evaluado mediante TCCB el grosor y altura del hueso alveolar a nivel de los dientes incisivos centrales y laterales. Sin embargo, la región canina y premolar maxilar también requiere de una rehabilitación estética, con parámetros anatómicos estables para planificar tratamietos o evaluar la estabilidad ósea a través del tiempo, pero relativamente pocos estudios se han realizado, los que se enfocan en el grosor óseo alrederor del Canino, 1PM y 2PM (Braut et al., 2011; Januário et al., 2012). 
Tabla I. Características morfométricas de la zona anterior de la maxila estudiada. Valores en mm, con un 95\% de confiabilidad.

\begin{tabular}{|c|c|c|c|c|c|c|}
\hline Hemiarco & Trazo & Diente & Media & $\pm \mathrm{DE}$ & Límite & Límite Sup. \\
\hline \multirow{11}{*}{ Derecho } & A-P & Canino & 17,72 & 0,63 & 16,45 & 18,99 \\
\hline & A-P & $1 \mathrm{e}^{\mathrm{r}} \mathrm{P}_{\text {remolar }}$ & 16,92 & 0,32 & 16,26 & 17,58 \\
\hline & A-P & 2do Premolar & $15,87^{*}$ & 3,83 & 15,17 & 16,57 \\
\hline & $\mathrm{R}-\mathrm{A}$ & Canino & 12,27 & 0,23 & 11,79 & 12,74 \\
\hline & $\mathrm{R}-\mathrm{A}$ & $1 \mathrm{e}^{\mathrm{r}} \mathrm{r}_{\text {remolar }}$ & 11,75 & 0,11 & 11,51 & 11,98 \\
\hline & $\mathrm{R}-\mathrm{A}$ & 2do Premolar & 11,57 & 0,26 & 11,03 & 12,11 \\
\hline & R-P & Canino & 26,22 & 0,87 & 24,46 & 27,99 \\
\hline & R-P & $1 \mathrm{e}^{\mathrm{r}} \mathrm{P}_{\text {remolar }}$ & 22,32 & 0,54 & 21,22 & 23,42 \\
\hline & R-P & 2do Premolar & 13,54 & 0,30 & 12,93 & 14,16 \\
\hline & LAC-A & Canino & $18,19^{*}$ & 3,05 & 12,05 & 24,32 \\
\hline & LAC-A & $1 \mathrm{e}^{\mathrm{r}} \mathrm{r}_{\text {remolar }}$ & 13,45 & 0,13 & 13,17 & 13,73 \\
\hline \multirow{13}{*}{ Izquierdo } & LAC-A & $1 \mathrm{e}^{\mathrm{r}} \mathrm{r}_{\text {remolar }}$ & 12,95 & 0,20 & 12,53 & 13,37 \\
\hline & A-P & Canino & $16,36^{*}$ & 0,59 & 15,18 & 17,55 \\
\hline & A-P & $1 \mathrm{e}^{\mathrm{r}} \mathrm{P}_{\text {remolar }}$ & 15,11 & 0,36 & 14,37 & 15,84 \\
\hline & A-P & 2do Premolar & 12,55 & 0,40 & 11,73 & 13,37 \\
\hline & $\mathrm{R}-\mathrm{A}$ & Canino & 11,90 & 0,23 & 11,41 & 12,38 \\
\hline & $\mathrm{R}-\mathrm{A}$ & $1 \mathrm{e}^{\mathrm{r}} \mathrm{P}_{\text {remolar }}$ & $11,58^{*}$ & 0,17 & 11,21 & 11,94 \\
\hline & $\mathrm{R}-\mathrm{A}$ & 2do Premolar & $10,43^{*}$ & 0,19 & 10,03 & 10,83 \\
\hline & $\mathrm{R}-\mathrm{P}$ & Canino & 33,88 & 4,7 & 24,42 & 43,33 \\
\hline & R-P & $1 \mathrm{e}^{\mathrm{r}} \mathrm{P}_{\text {remolar }}$ & 25,74 & 0,74 & 24,24 & 27,24 \\
\hline & $\mathrm{R}-\mathrm{P}$ & 2do Premolar & 14,65 & 0,69 & 13,24 & 16,05 \\
\hline & LAC-A & Canino & 13,06 & 0,10 & 12,86 & 13,27 \\
\hline & LAC-A & $1 \mathrm{e}^{\mathrm{r}} \mathrm{P}_{\text {remolar }}$ & 12,68 & 0,14 & 12,39 & 12,97 \\
\hline & LAC-A & $1 \mathrm{e}^{\mathrm{r}} \mathrm{P}_{\text {remolar }}$ & 12,29 & 0,19 & 11,90 & 12,68 \\
\hline
\end{tabular}

$\mathrm{DE}=$ Desviación estándar; A-P= unión del punto más apical del diente a medir (A) y punto localizado a nivel de la pared inferior del seno maxilar (P); $\mathrm{R}-\mathrm{A}=$ unión del punto localizado por distal de los dientes y a nivel del reborde alveolar $(\mathrm{R})$ y punto A; R-P= unión de punto R y P; LAC-A= unión del punto localizado en la zona de unión cemento esmalte de los dientes y por distal de estos (LAC) y A.

Considerando que la literatura ha mostrado que menos de $10 \mathrm{~mm}$ de altura ósea puede no ser adecuada para el éxito de implantes dentales a largo plazo (Esposito et al., 2009), los valores del trazo R-P (por ejemplo en el 2PM correspondiente a 13,54 $\mathrm{mm}$ en el hemiarco derecho y 14,65 $\mathrm{mm}$ en el izquierdo), adquiere especial relevancia en la preservación del reborde alveolar tras la exodoncia, lo que concuerda con investigaciones realizadas por Araujo et al. (2005), quienes describen alteraciones dimensionales importantes posterior a la extracción de premolares debido a una marcada actividad osteoclástica, que trae como resultado la resorción del reborde alveolar. La reducción de altura ósea, excluyendo accidentes como fractura de la tabla ósea bucal, es acompañada por una pérdida de horizontal de la misma (Araujo \& Lindhe). Januário et al. informaron cambios en las dimensiones de la pared ósea en la zona anterior del maxilar (incisivo central, incisivo lateral y canino) mediante TCCB, principalmente el espesor medio de la tabla ósea bucal a nivel del canino a los 1, 3 y $5 \mathrm{~mm}$ apical a la cresta ósea, diminuyendo a menos de $1 \mathrm{~mm}$, lo que también ocurre a nivel de los premolares.

Los datos aportados por el presente estudio, relacionaron en un $80 \%$ al canino en una posición mesial a la tangente a la pared anterior del seno (T). Estas relaciones ana- tómicas son relevantes al momento de la planificación de tratamientos mediante implantes dentales u otras cirugías que involucren el abordaje de la zona. Chiapasco \& Zaniboni (2009); de Moraes et al. (2012) y Mendoza et al. (2013) describen relaciones del reborde y los dientes en la zona posterior y piso del seno maxilar, pero no encontramos otros reportes utilizando TCCB relacionando la zona anterior maxilar alrededor de los caninos y premolares.

Estos resultados se deben considerar antes de la extracción del diente y la planificación de la rehabilitación en la zona canina y premolar del maxilar. Un análisis preoperatorio cuidadoso mediante TCCB puede ser beneficioso en el diagnóstico para evaluar los factores de riesgo locales y para lograr una alta predictibilidad.

FUENTES, R.; NAVARRO, P.; SALAMANCA, C.; CANTÍN, M.; GARAY, I. \& FLORES, T. Morphometric characterization of anterior maxillary alveolar ridge by Cone-Beam computed tomography. Int. J. Morphol., 32(2):493-498, 2014.

SUMMARY: The images obtained by Cone -Beam Computed Tomography (CBCT) allow the determination of threedimensional relationships of anatomical structures and overcome limitations of conventional radiography. The aim of this study was 
to describe the morphometric characteristics of the anterior maxilla and its relation to canine and premolars by CBCT exams. A descriptive study on $50 \mathrm{CBCT}$ images was made, following a systematic observation of three-dimensional anatomical points between teeth and maxillary sinus walls, they were located and plotted tangent for measurement. The Mean and SD was calculated for each parameter, and the normality of distribution was tested by Kolmogorov -Smirnov test. Data were compared by ANOVA test, with a $p$ value $<0.05$ as significant. The analysis of the relationship between the teeth and the anterior wall of the maxillary sinus, showed that in $80 \%$ of cases are associated with the canine and in $20 \%$ to first molar. The lower height between the alveolar ridge and the inferior wall of the maxillary sinus was observed at the second premolar with $13.54 \pm 0.30 \mathrm{~mm}$ on the right side and $14.65 \pm 0.30 \mathrm{~mm}$ on the left. Significant differences were recorded in relation to morphometric measurements and age range variable. The data obtained in this study complement the morphometric characteristics of the anterior maxilla, allowing implant treatments in a scientific morphological basis and minimizing the level of risk of failure or damage to the anatomical structures related.

KEYWORDS: Maxillary sinus; Dental implants; Tooth canine; Maxillary first premolar.

\section{REFERENCIAS BIBLIOGRÁFICAS}

Arana-Fernández, E.; Buitrago-Vera, P.; Benet-Iranzo, F. \& TobarraPérez, E. Evaluación de la Tomografía Computarizada: Introducción a las aplicaciones dentales. RCOE, 11(3):311-22, 2006.

Araújo, M. G. \& Lindhe, J. Dimensional ridge alterations following tooth extraction. An experimental study in the dog. J. Clin. Periodontol., 32(2):212-8, 2005.

Ausbruch, C.; Golco, G. \& Sirimarco, F. Comparación milimétrica de los diversos métodos radiográficos para el diagnóstico preimplantológico: Estudio comparativo de 40 casos. Rev. Círc. Argent. Odontol., 67(209):6-12, 2010.

Barrachina-Mataix, M.; Cabello-Domínguez, G.; Olmos-Sanz, G. \& González-Fernández D. Tratamiento implantológico de la zona posterior del maxilar superior. Elevación del seno maxilar. RCOE, 7(1):91-100, 2002.

Bergkvist, G.; Sahlholm, S.; Karlsson, U.; Nilner, K. \& Lindh, C. Immediately loaded implants supporting fixed prostheses in the edentulous maxilla: a preliminary clinical and radiologic report. Int. J. Oral Maxillofac. Implants, 20(3):399-405, 2005.

Braut, V.; Bornstein, M. M.; Belser, U. \& Buser, D. Thickness of the anterior maxillary facial bone wall-a retrospective radiographic study using cone beam computed tomography. Int. J. Periodontics Restorative Dent., 31(2):125-31, 2011.

Chiapasco, M. \& Zaniboni, M. Methods to treat the edentulous posterior maxilla: implants with sinus grafting. J. Oral Maxillofac. Surg., 67(4):867-71, 2009.
Esposito, M.; Grusovin, M. G.; Felice, P.; Karatzopoulos, G.; Worthington, H. V. \& Coulthard, P. The efficacy of horizontal and vertical bone augmentation procedures for dental implants - a Cochrane systematic review. Eur. J. Oral Implantol., 2(3):167-84, 2009.

Figún, E. \& Garino, R. Anatomía odontológica: funcional y aplicada. $2^{\mathrm{a}}$ ed. Buenos Aires, El Ateneo, 2002. pp.234-40.

Fuentes, F. R.; Borie, E. E.; Bustos, M. L. \& Thomas, M. D. First premolar morphometry: A study in 54 cases. Int. J. Morphol., 29(1):234-9, 2011.

Januário, A. L.; Duarte, W. R.; Barriviera, M.; Mesti, J. C.; Araújo, M. G. \& Lindhe, J. Dimension of the facial bone wall in the anterior maxilla: a cone-beam computed tomography study. Clin. Oral Implants Res., 22(10):1168-71, 2011.

Leblebicioglu, B.; Rawal, S. \& Mariotti, A. A review of the functional and esthetic requirements for dental implants. J. Am. Dent. Assoc., 138(3):321-9, 2007.

Mantini, S. \& Ripani, M. Modern morphometry: new perspectives in physical anthropology. N. Biotechnol., 25(5):325-30, 2009.

de Moraes, P. H.; Costa, V. O. C.; Olate, S.; Caria, P. H. F. \& Barbosa, J. R. A. Estudio morfométrico del seno maxilar mediante tomografías computadorizadas. Evaluación de la reconstrucción total de piso sinusal. Int. J. Morphol., 30(2):592-598, 2012.

Mendoza, G.; Morales, P.; Reyes, F.; Navarro, P.; Garay, I. \& Olate, S. The alveolar bone high in posterior area of maxilla and the relation with tooth loss. Int. J. Morphol., 31(3):822-5, 2013.

Mozzo, P.; Procacci, C.; Tacconi, A.; Martini, P. T. \& Andreis, I. A. A new volumetric $\mathrm{CT}$ machine for dental imaging based on the conebeam technique: preliminary results. Eur. Radiol., 8(9):1558-64, 1998.

Nothdurft, F. P.; Seidel, E.; Gebhart, F.; Naumann, M.; Motter, P. J. \& Pospiech, P. R. The fracture behavior of premolar teeth with class II cavities restored by both direct composite restorations and endodontic post systems. J. Dent., 36(6):444-9, 2008.

Oviedo, P. \& Hernández, J. Tomografía computarizada Cone Beam en endodoncia. Rev. Estomatol. Herediana, 22(1):59-64, 2012.

Swart, L. C. \& van Niekerk, D. J. Simplifying the implant treatment for an unrestorable premolar with a one-piece implant: a clinical report. J. Prosthet. Dent., 100(2):81-5, 2008.

Dirección para Correspondencia:

Prof. Dr. Ramón Fuentes Fernández

Departmento Odontología Integral

Facultad de Odontología

Universidad de la Frontera

Manuel Montt 112,Temuco

CHILE

Recibido : 09-01-2014

Aceptado: 16-03-2014

Email: ramon.fuentes@ufrontera.cl 